\title{
Characterization and Reactivity of Sodium Aluminoborosilicate Glass Fiber Surfaces
}

Lymaris Ortiz Rivera ${ }^{1}$, Victor Bakaev ${ }^{1}$, Joy Banerjee ${ }^{1}$, Karl T. Mueller ${ }^{2}$ and Carlo G. Pantano ${ }^{1,3}$

Materials Research Institute ${ }^{1}$, Department of Chemistry² and Department of Materials Science and Engineering ${ }^{3}$, Pennsylvania State University, University Park, Pennsylvania, 16802, USA

\begin{abstract}
Multicomponent complex oxides, such as sodium aluminoborosilicate glass fibers, are important materials used for thermal insulation in buildings and homes. Although the surface properties of single oxides, such as silica, have been extensively studied, less is known about the distribution of reactive sites at the surface of multicomponent oxides. Here, we investigated the reactivity of sodium aluminoborosilicate glass fiber surfaces for better understanding of their interface chemistry and bonding with acrylic polymers. Acetic acid (with and without a ${ }^{13} \mathrm{C}$ enrichment) was used as a probe representative of the carboxylic functional groups in many acrylic polymers and adhesives. Inverse gas chromatography coupled to a mass spectrometer (IGC-MS), and solid state nuclear magnetic resonance (NMR), were used to characterize the fiber surface reactions and surface chemical structure. In this way, we discovered that both sodium ions in the glass surface, as well as sodium carbonate salts that formed on the surface due to the intrinsic reactivity of this glass in humid air, are primary sites of interaction with the carboxylic acid. Surface analysis by X-ray photoelectron spectroscopy (XPS) confirmed the presence of sodium carbonates on these surfaces. Computer simulations of the interactions between the reactive sites on the glass fiber surface with acetic acid were performed to evaluate energetically favorable reactions. The adsorption reactions with sodium in the glass structure provide adhesive bonding sites, whereas the reaction with the sodium carbonate consumes the acid to form sodium-carboxylate, $\mathrm{H}_{2} \mathrm{O}$ and $\mathrm{CO}_{2}$ without any contribution to chemical bonding at the interface.
\end{abstract}

\section{Introduction}

The applications of glass fibers are diverse; they are used for insulation, filtration, reinforcements and more [1]. Chemical knowledge obtained from single oxide materials is often applied to understand multicomponent oxide surfaces, even though it is widely recognized that a broad distribution of reactive sites exist at the surface of these more complex systems [2-3]. The surface atomic structure of sodium aluminoborosilicate glass fibers is heterogeneous due to its disorder and the multicomponent nature of this glass system. Thus, a wide distribution of adsorption sites can be expected. In the case of these hydrolytically reactive alkali-silica surfaces, reactions with humidity can further modify the surface. Of particular relevance here is the formation of carbonate salts on the surface during processing and storage due to atmospheric weathering [4-8]. This can deplete the alkali through an ion exchange reaction with water to create a hydrated surface layer and sodium-carbonate reaction products.

Understanding the interfacial bonding of a polymer with a glass surface requires a thorough knowledge of the reactive sites at the polymer/glass interface. The specific bonds that form when multicomponent aluminoborosilicate glasses are dosed with simple organic molecules (representative of the functional groups in the polymer) is one way to understand these surface reactions and the interface they produce. In a previous study [10], the adsorption of acetic acid was determined to be very different on this sodium aluminoborosilicate glass compared to a pure silica surface. Whereas silyl-ester bonds linked the acetic acid to the pure silica surface, the formation of 
sodium-carboxylate was observed on the sodium-aluminoborosilicate surface. It was not clear whether the carboxylate was created as a distinct phase via leaching of sodium from the glass surface, or formed a complex with sodium ions within the glass surface structure. The possible effects of surface hydration and weathering were not considered, although in most industrial practices there is limited exposure of the fresh fiber surface to humidity before the coating is applied. In this study, the surface reactions and chemical structure of the reaction products on the multicomponent glass fibers are examined in more detail using a combination of inverse gas chromatography with a mass-spectrometer (IGC-MS) and solid-state nuclear magnetic resonance (NMR). We also use the deconvolution of a NMR line to refine the conclusion of previous NMR studies [10]. Geometry optimization using Gaussian 03 software package was used to study the thermodynamic favorability of different reaction complexes. Acetic acid was chosen as the probe molecule due to the prevalence of carboxylic acid groups in many commercial polymer adhesives and coatings, and their potential to form silyl ester linkages to the silicate network and carboxylates with sodium (and other alkali or alkaline earth species in glass).

The term IGC unifies several chromatographic techniques that, in contrast with conventional chromatography, are used not for the study of a mobile phase but for the study of a stationary phase, which in this case is a bundle of glass fibers. IGC is mainly used for measuring surface energies (IGC at infinite dilution), adsorption isotherms (IGC at finite concentrations) and diffusion in polymers among others [11]. However, in this study IGC-MS is used to study chemical reactions on glass fiber surfaces.

\section{Experimental Section \\ 2.1 Materials}

Sodium aluminoborosilicate glass fibers were drawn using a lab-scale single-tip fiber drawing system. Bulk glass marbles (a commercial glass wool insulation composition, courtesy of JohnsManville) were re-melted at $1000{ }^{\circ} \mathrm{C}$ in a single tip platinum-rhodium crucible and allowed to equilibrate for 1 hour before drawing fiber. The melted glass was drawn through a $2 \mathrm{~mm}$ diameter tip at $4.6 \mathrm{~m} / \mathrm{s}$ onto a rotating Teflon cylinder. Fibers were drawn for 15 minutes under standard laboratory conditions $\left(25^{\circ} \mathrm{C}\right.$ and less than $25 \%$ relative humidity) and either analyzed immediately or stored under vacuum for future analyses. Fibers had an average diameter of approximately $24 \mathrm{~m}$.

Analytical columns for the IGC experiments consisted of quartz tubing (6.3 $\mathrm{mm}$ outer diameter, $4 \mathrm{~mm}$ inner diameter, Technical Glass Products) with a length of $23 \mathrm{~cm}$. The quartz tubing was cleaned using ethanol and then dried in a vacuum oven for 12 hours at $125^{\circ} \mathrm{C}$. Fibers were packed into the column by tying wax-free dental floss around the center of a $52 \mathrm{~cm}$ long monofilament bundle. The strand of floss was then used to pull the fibers through the column ensuring alignment of the fibers along the column axis. The column was packed as densely as possible to maximize the amount of surface area available for adsorption. On average, $3 \mathrm{~g}$ of fibers could be packed within the $23 \mathrm{~cm}$ long, $4 \mathrm{~mm}$ internal diameter column.

Acetic acid (HPLC grade, $>99 \%$, Sigma Aldrich) and isotopically enriched acetic acid ${ }^{13} \mathrm{C}$ (99 atom \%, Sigma-Aldrich) were used for the adsorption experiments. Sodium carbonate (ACS primary standard, 99.95-100.05\% (dried basis), Alfa Aesar) was used for the NMR experiments. 


\subsection{Characterization Methods \\ 2.2.1 IGC-MS}

IGC-MS experiments were performed using a standard GC (gas chromatograph) (HP 5890 Series II GC) equipped with a 5792 mass spectrometer (GC-MS). The oven temperature was set at $100^{\circ} \mathrm{C}$ and the split/splitless injector temperature was set at $60^{\circ} \mathrm{C}$. A cup liner was used with injection directly in the cup; this created a broad peak of solute concentration at the inlet of the column. The pressure drop on the packed column (a fraction of a psi) is too small for the electronic pressure control (EPC) of the HP 5890. For that reason, the deactivated guard column (6 m length, $0.25 \mathrm{~mm}$ internal diameter) was inserted between the injector and the packed column.

Once the column is loaded in the GC, it is conditioned under UHP (Ultra High Purity) helium $(10 \mathrm{ml} / \mathrm{min})$ at $200{ }^{\circ} \mathrm{C}$ for 30 minutes before any experiment begins. A typical IGC analysis consisted of an injection of $1 \mathrm{~L}$ of acetic acid using a Hamilton syringe (7000 series, Hamilton Company, Reno, NV). Before injection the mass sensitive detector (MSD) is turned on and a time delay of 0.5 minutes precedes the actual injection.

A GC/MS chromatogram consists of separate points, each point containing the entire mass spectrum. The mass spectra were scanned in the interval of $\mathrm{m} / \mathrm{z}$ values from 10 to $70 \mathrm{Da}$. There are two standard procedures the MS software allows one to carry out with those spectra: background subtraction and averaging. Both were employed in this study. The background spectrum was recorded during the first $0.5 \mathrm{~min}$ before injection of acetic acid and was subtracted from all the target spectra. The background spectrum included mainly the lines of $\mathrm{m} / \mathrm{z}=17,18$ (water) and $\mathrm{m} / \mathrm{z}=28,32$ (air). Averaging allows one to average spectra in a predetermined area, it is a process performed by the instrument software. In this study averaged areas are the portions within the lines marked in the spectra (Figure 3). Averaging allows to have a better representation of the masses obtained in a certain region and it is based on time, not $\mathrm{m} / \mathrm{z}$.

Another subtraction procedure used in this work was subtraction of the pure acetic acid spectrum from the sample spectrum. The goal of this subtraction was to discover the reaction products which appear due to interaction of acetic acid with the glass surface. The pure acetic acid subtraction is performed using a special Microsoft Excel module developed for this work. First, the mass spectrum of pure acetic acid is obtained without the packed column (the guard column is connected to the MS). The three lines with highest abundance in this spectrum are selected, and then the same three lines are searched for in the target spectrum. A ratio of abundance for each of the three lines in the target and pure acetic acid spectra is made, and an average is calculated. Then, all the lines in the pure acetic acid spectra are multiplied by that averaged ratio and subtracted from the target spectrum.

There are three molecules (carbon dioxide, water and acetic acid) whose mass spectra are discussed below. Those mass spectra can be found in [12].

\subsubsection{Nuclear Magnetic Resonance (NMR)}

For NMR experiments, glass fibers and sodium carbonate powder samples were packed into 4 $\mathrm{mm}$ (outer diameter) zirconia rotors and fitted with a Kel-F drive cap. Sample preparation, including the packing and unpacking of rotors, was performed under an inert nitrogen atmosphere utilizing a

glove bag apparatus. The NMR probe molecule was ${ }^{13} \mathrm{C}$ carbonyl enriched (99 atom \%) acetic acid (Sigma-Aldrich, St. Louis, MO). 
A custom built dosing apparatus was used to perform vapor dosing of NMR samples. The apparatus consisted of a roughing pump connected to a glass manifold with two separate chambers. One of the chambers, called the sample chamber, was heated using an external furnace, and the second chamber held acetic acid. The dosing experiment consist by loading about $0.5 \mathrm{~g}$ of sample and then heating the sample to $125{ }^{\circ} \mathrm{C}$ for $30 \mathrm{~min}$ under vacuum $(\sim 1 \times 10-3$ Torr $)$. After the 30 minutes of heating, the vacuum valve was closed, and the dosing chamber is opened to expose the sample to the vapor of acetic acid for up to $16 \mathrm{~h}$. After dosing, the sample chamber was disconnected from the apparatus and placed in a $\mathrm{N}_{2}$ purged glove bag in order for the sample to be packed into NMR rotors. All sample preparation including the packing and unpacking of rotors was performed under an inert nitrogen atmosphere utilizing a glove bag.

${ }^{1} \mathrm{H}-{ }^{13} \mathrm{C}$ cross polarization (CP) magic-angle spinning (MAS) NMR experiments were performed with high-power proton decoupling on a Bruker 7.0 Tesla NMR Spectrometer operating at the ${ }^{13} \mathrm{C}$ irradiation frequency of $75.551 \mathrm{MHz}$ and a ${ }^{1} \mathrm{H}$ frequency of $300.431 \mathrm{MHz}$. Experimental setup was performed using Bruker's software package (Topspin version 1.3) and the NMR console was interfaced to a Bruker $4 \mathrm{~mm}$ double-resonance (DR) wide bore MAS probe. All experiments acquired 16,000 transients with a total of 4096 complex points and $40 \mathrm{kHz}$ sweep width. CP MAS was achieved utilizing a ${ }^{1} \mathrm{H} \pi / 2$ pulse width of $5.18 \mu \mathrm{s}$, contact time of $5 \mathrm{~ms}$, recycle delay of $5 \mathrm{~s}$, and a MAS spinning speed of $5000 \mathrm{~Hz}$.

NMR decoupling was performed using NUTS software (Acorn NMR Inc., Livermore, CA).

\subsubsection{X-Ray Photoelectron Spectroscopy (XPS)}

A Kratos Analytical Axis Ultra spectrometer (Chestnut, NY) fitted with a monochromatic AlK $\alpha(1486.6 \mathrm{eV}) \mathrm{X}$-ray source was used to characterize the samples. To minimize charging of the surface, samples were packed in an aluminum foil packet and mounted with double-sided tape with a low-energy electron flood gun employed during acquisition. Survey scans for the quantification of $F$ 1s, O 1s, Na KLL, Ca 2p, Mg KLL, K 2p, C 1s, B 1s, Si 2p and Al 2p peaks were conducted at 80 $\mathrm{eV}$ pass energy with step size of $0.3 \mathrm{eV}$ and dwell time of $150 \mathrm{~ms}$ in hybrid mode. Sufficient peak to noise ratio were present for the curve fitting of $\mathrm{C} 1 \mathrm{~s}$ photoelectron peak in the survey spectrum to determine the chemical states of carbon. Curve-fitting of the $\mathrm{C} 1 \mathrm{~s}$ spectrum and quantification of the survey spectrum to obtain elemental concentration were conducted with the CASA XPS software package [13].

Figure 1 shows a representative spectra of the carbon $1 \mathrm{~s}(\mathrm{C} 1 \mathrm{~s})$ peak. The $\mathrm{C} 1 \mathrm{~s}$ peak is fitted with component peaks using the Voigt line shapes GL35 (65\% Gaussian, 35\% Lorentzian) [14]. In the case of the $\mathrm{C}$ 1s spectrum depicted in Figure 1, four component peaks are required to fit the main peak with a residual value of less than 1.0 [15]. The component peaks are unconstrained with respect to peak position and FWHM (full width half max) prior to fitting with the Marquardt algorithm. The four peaks (in order of increasing binding energy) are attributed to hydrocarbon species of alkyl (C$\mathrm{C})$, carbonyl (C-O) and acid (-COOH) [12], and carbonate (-CO3) [16]. The carbon's alkyl (C-C/H) peak is assigned binding energy of $284.6 \mathrm{eV}$, and the survey spectrum and other $\mathrm{C} 1 \mathrm{~s}$ component peaks are shifted accordingly prior to quantification. Fractional carbon speciation is obtained by normalization of the area under each component peak to the area of the main $\mathrm{C}$ 1s peak envelope. Elemental composition is determined by the ratio of the single signature peak of each element normalized by the relative sensitivity factor (RSF) and transmission function of the instrument, to the total sum of areas of signature elemental photoelectron peaks with the same normalization. The RSF's for the instrument were determined with reference materials such as in-vacuo fracture surface 
of the bulk sodium-aluminoborosilicate glass for the elements in the glass and polydimethylsiloxane (PDMS) for carbon. For this study, it is more important to identify the presence of carbonates than obtain the precise quantity of carbonate species. Therefore, the RSF of carbon is deemed sufficient to quantify the carbon speciation in terms of atomic \%. The error associated with the elemental composition was determined to be less than $10 \%$ by multiple sampling.

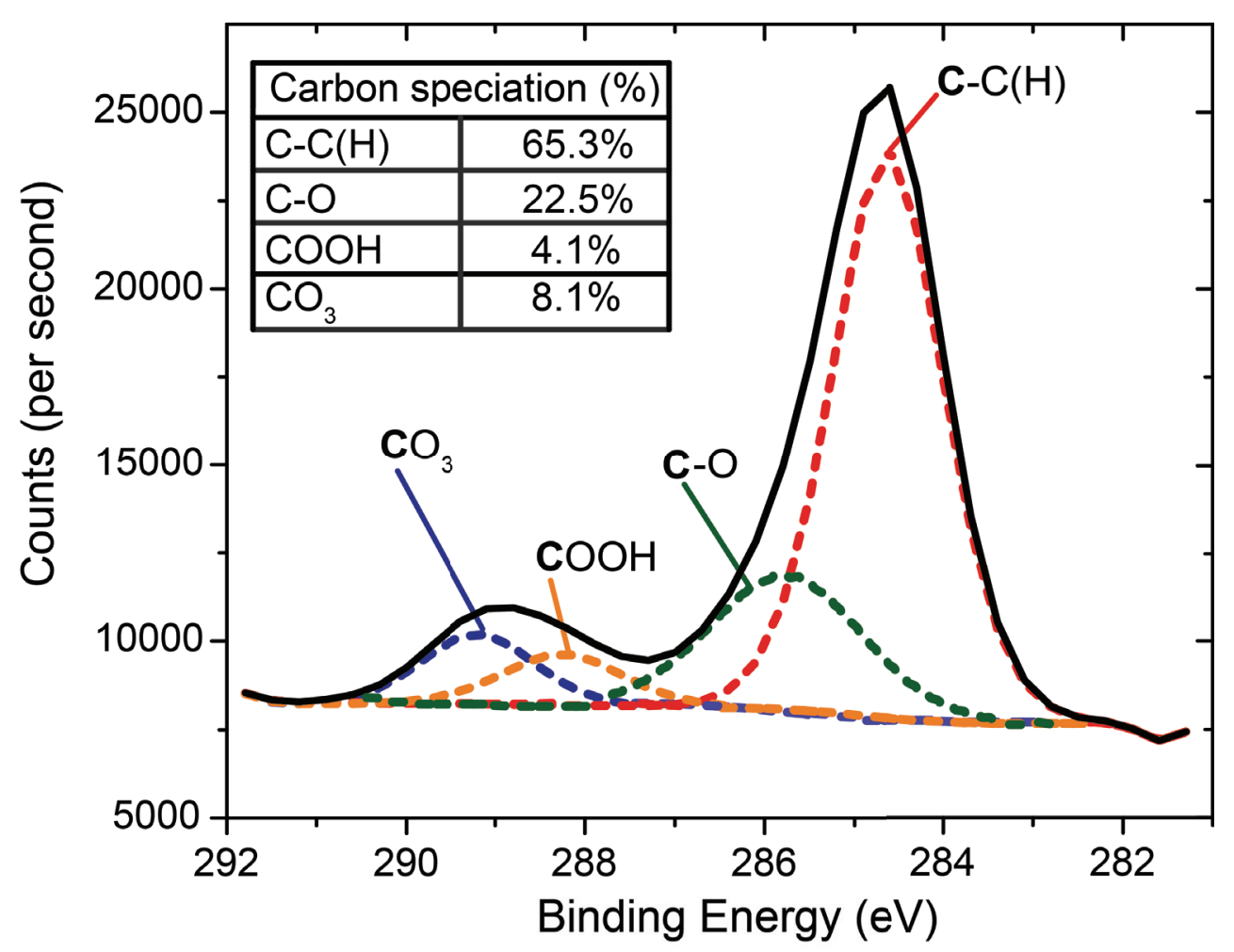

Figure 1. C 1s spectrum of sodium aluminoborosilicate glass fiber.

\subsubsection{Computer Simulation}

Cursory computer simulations were performed based on Density Functional Theory (DFT) [17], taking into account all the electrons, and using the combination of Becke's three parameter exchange functional and Lee, Yang and Parr's correction to the local correlation potential (B3LYP) $[18,19]$, as implemented in the Gaussian 03 software package[20]. A $6-311++\mathrm{g}(\mathrm{d}, \mathrm{p})$ split valence basis set including polarization functions and diffuse functions for heavy atoms were employed. The use of this basis set was deemed appropriate because it has already been used to model the carboxylic acid-based molecules of interest elsewhere [21,22]. The molecular and glass surface clusters were formed by positioning atoms with bond distances found in literature and then performing iterative calculations to find the geometry with the lowest energy configuration. The geometry optimization utilizes the Berny algorithm with Self Consistent Field (SCF) cycles with a convergence threshold of 1E-8 Hartree and random mean square (RMS) force threshold of 3E-4 Hartree/Å. Zero point energy (ZPE) and basis set superposition error (BSSE) correction for the structures in this study is not implemented. 
The energy of interaction $\left(\Delta \mathrm{E}_{\text {int }}\right)$ between the adsorbate species and the glass surface cluster was then calculated by subtracting the binding energy $(\mathrm{BE})$ of the optimized reactants from the optimized product (Equation 1) [23]. If the energy of the product is less than the reactants $\left(\Delta \mathrm{E}_{\mathrm{int}}<0\right)$, the interaction between the two groups is considered to be thermodynamically favorable.

$$
\Delta E_{\text {int }}=B E(\text { product })-B E(\text { reactants })
$$

\section{Results and Discussion}

Table 1 presents the elemental surface composition, and Figure 2 shows a typical survey spectrum, of the glass fibers. The quantified carbon speciation ( $\underline{\mathrm{C}}-\mathrm{C}, \underline{\mathrm{C}}-\mathrm{O}, \underline{\mathrm{COOH}}$ and $\underline{\mathrm{CO}} \mathrm{O}_{3}$ ) is obtained by multiplying the fraction of speciation by the determined carbon content in atomic concentration. We assume that the adventitious surface hydrocarbons $(\mathrm{C}-\mathrm{C}, \mathrm{C}-\mathrm{O}$ and $\mathrm{COOH}$ ) will desorb during the initial outgassing of the fiber samples in the IGC at $100^{\circ} \mathrm{C}$. But the $\mathrm{C} 1 \mathrm{~s}$ spectrum also reveals that $\sim 0.8$ atomic $\%$ of the carbon is in the form of carbonate. Based on XPS data in Table 1, sodium is the most favorable modifier species for carbonate formation [24]. Assuming the stoichiometry of sodium carbonate, it can be inferred that $\sim 1.6$ atomic $\%$ of the $\mathrm{Na}$ is associated with the carbonate phase on the surface (crystals based on electron microscopy) and 8.2 atomic $\% \mathrm{Na}$ is retained in the surface of the glass. Thus XPS analysis suggests the presence of sodium carbonates on the fiber surface. The following IGC-MS and NMR studies refine that suggestion.

Table 1. XPS surface composition (in atomic \%) of the sodium aluminoborosilicate glass fibers.

\begin{tabular}{|c|c|c|c|c|c|c|c|c|c|c|c|}
\hline $\mathrm{O}$ & $\mathrm{B}$ & $\mathrm{Si}$ & $\mathrm{Al}$ & $\mathrm{Na}$ & $\mathrm{Ca}$ & $\mathrm{F}+\mathrm{Mg}+\mathrm{K}$ & $\mathrm{C}$ & $\mathrm{C}-\mathrm{C}$ & $\mathrm{C}-\mathrm{O}$ & $\mathrm{COOH}$ & $\mathrm{CO}_{3}$ \\
\hline 54.1 & 4.5 & 16.0 & 1.9 & 9.8 & 2.3 & 1.5 & 9.9 & 6.4 & 2.2 & 0.4 & 0.8 \\
\hline
\end{tabular}




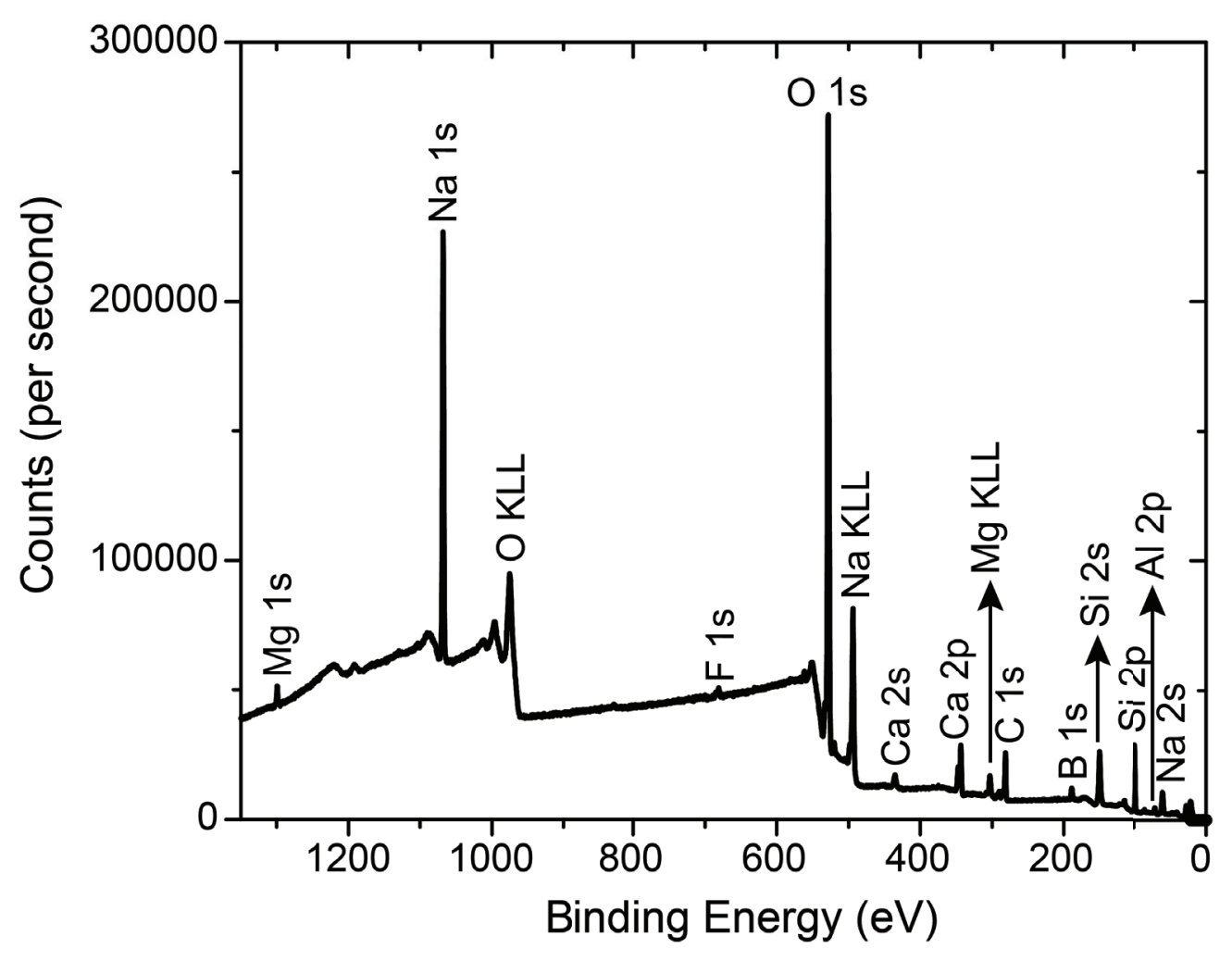

Figure 2. XPS survey spectrum of sodium aluminoborosilicate glass fiber.

The initial IGC adsorption experiments used acetic acid with natural isotopic abundance. Figure 3 shows the output of MSD in the form of a total ionic current (TIC) after a $1 \mu \mathrm{L}$ injection of acetic acid. It consists of one large broad peak which is preceded by two smaller overlapping peaks/shoulders at the beginning of the elution. The peaks are broad because the injection pulse is also broad. As noted above, each point in the MSD output corresponds to an entire mass spectrum. For our purposes here, we recognize three distinct events labeled A, B and C in Figure 3. For each of these regions, we have averaged (see Section 2.2.1) all of their belonging spectra; the averaged spectra are presented in Figure 4. 


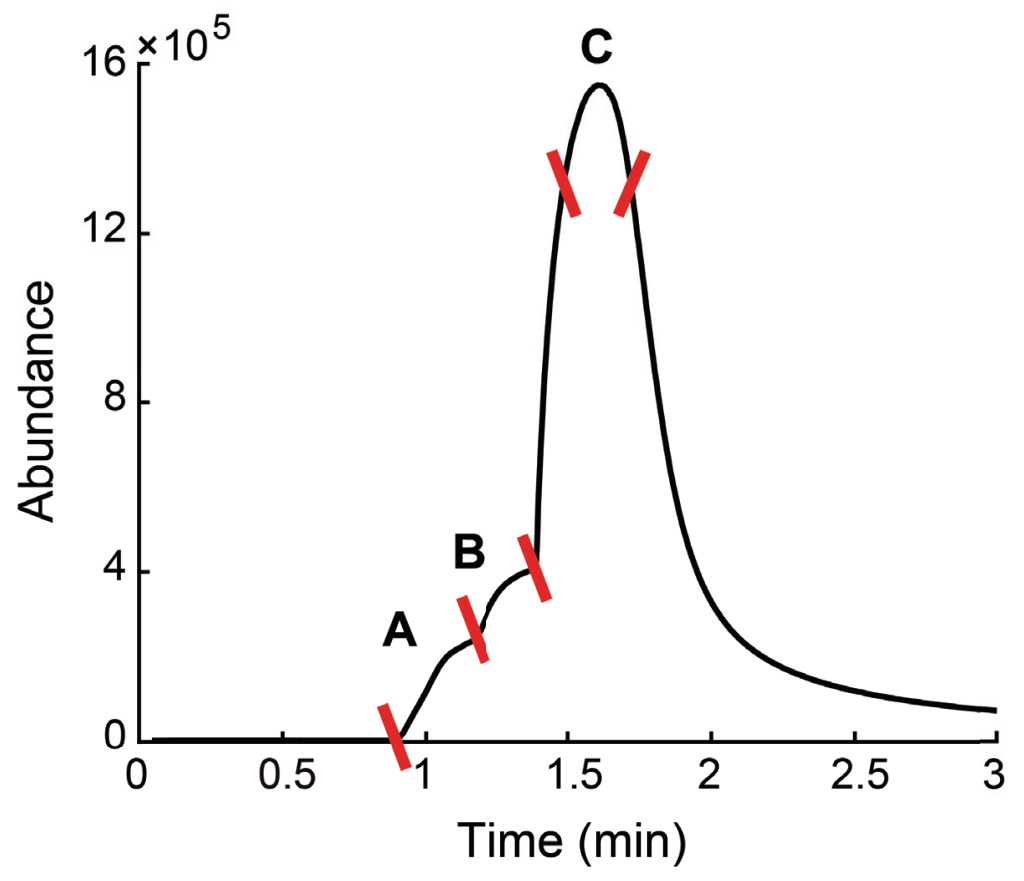

Figure 3. IGC-MS of sodium aluminoborosilicate glass fiber dosed with acetic acid at $100{ }^{\circ} \mathrm{C}$. Lines indicate borders of averaging.

A

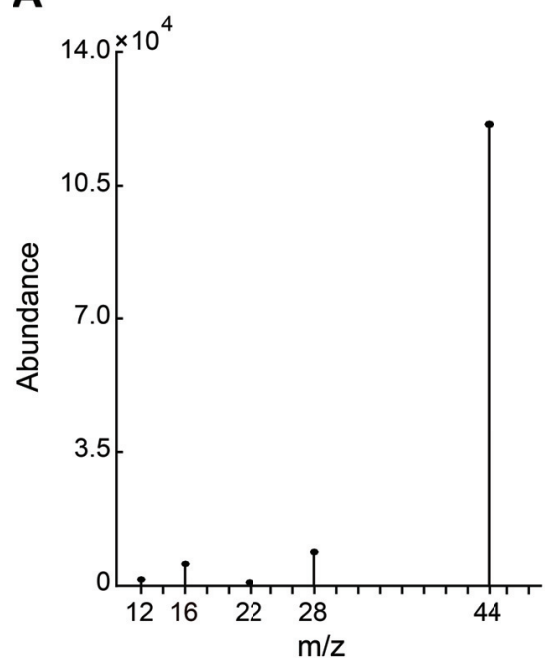

B

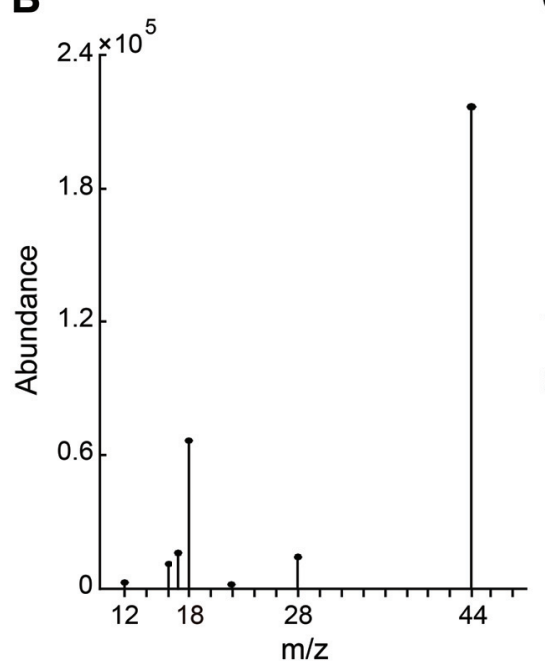

C

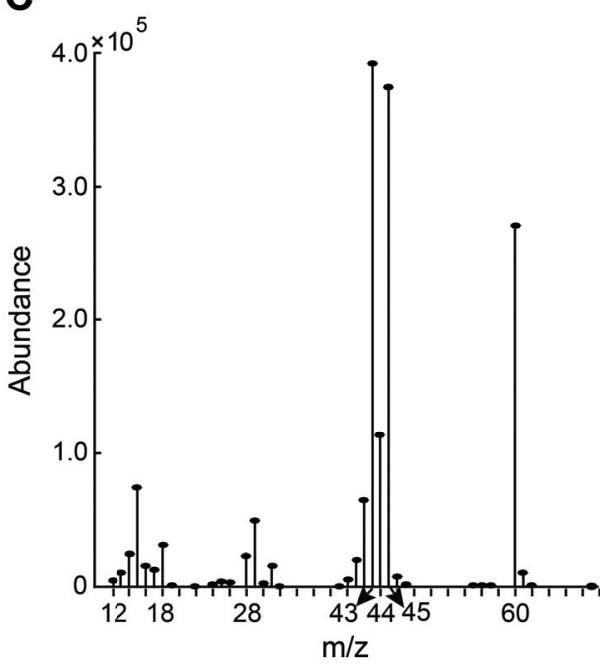

Figure 4. Mass spectra averaged over the areas A, B and C in Figure 3. 


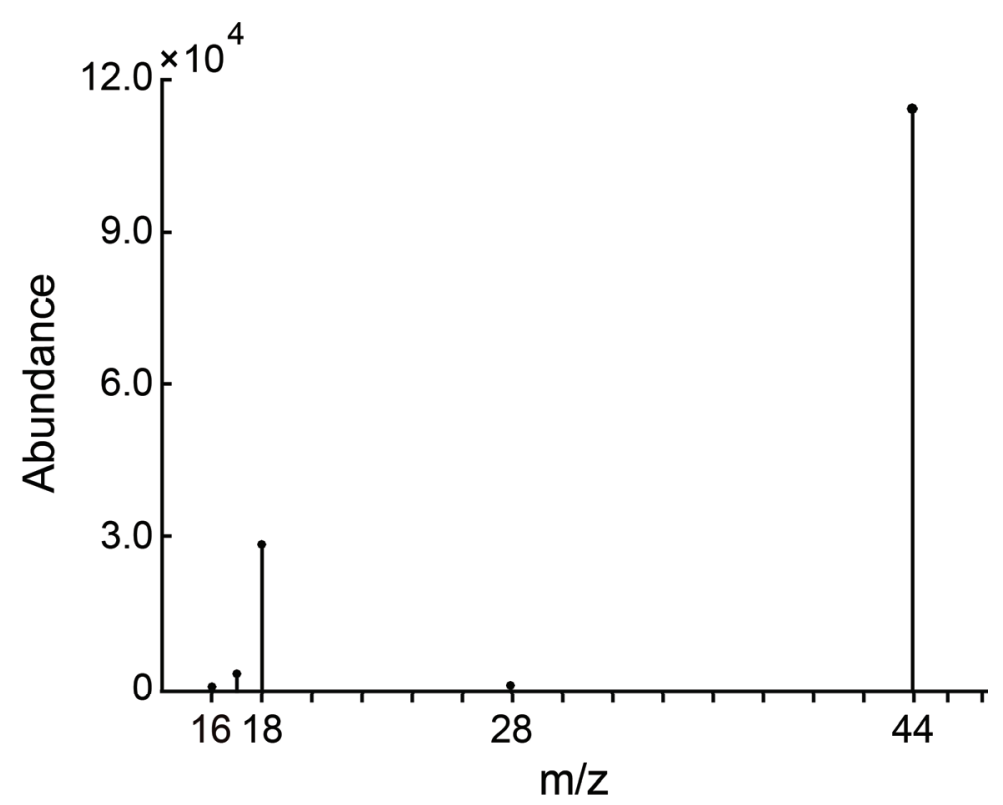

Figure 5. Residual spectrum after subtraction of the acetic acid spectra from Figure 4C (region C).

The averaged mass spectra of region A (Figure 4A) shows that the initial elution is composed primarily of species with $\mathrm{m} / \mathrm{z}$ of $12,16,22,28$ and 44 . This mass spectrum corresponds to carbon dioxide (see [12]). Considering the XPS detection of sodium carbonate on the fiber surfaces Fig. 1, this suggests that the most prevalent reaction initially occurs between the acetic acid and the sodium carbonate as a result of weathering of the fiber surface, as shown in Scheme 1. This reaction forms sodium-carboxylate resulting a release of carbon dioxide as observed in Figure 3,

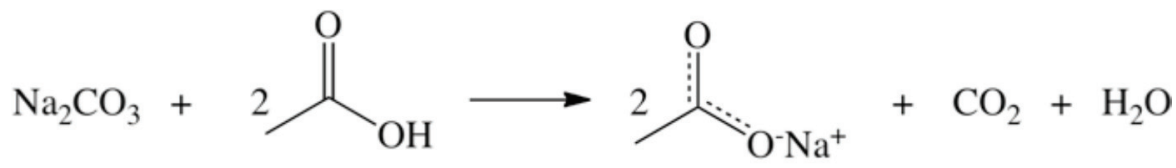

Scheme 1. Reaction between acetic acid and sodium carbonate weathering products on the fiber surface.

The averaged mass spectra for region B (of Figure 3) is presented in Figure 4B and shows elution of species with $\mathrm{m} / \mathrm{z}$ of $12,16,17,18,22,28$ and 44 . The species with $\mathrm{m} / \mathrm{z}$ equal to 17 and 18 correspond to water and the remaining species to carbon dioxide ( $\mathrm{m} / \mathrm{z}=15$ is uncompensated noise). The abundance of carbon dioxide in this region $\mathrm{B}$ shoulder is higher than that of water and higher than the abundance of carbon dioxide in the region A shoulder. The reaction of acetic acid with sodium carbonate produces water and carbon dioxide as depicted in the reaction above. But since the retention time of carbon dioxide at room temperature is much smaller than that of water, the carbon dioxide elutes faster than water to produce the two overlapping regions A and B in Figure 3.

The major peak in the chromatogram is region $\mathrm{C}$ at $\sim 1.5$ minutes (in Figure 3 ). It has a complicated mass spectrum with high abundance for species with $\mathrm{m} / \mathrm{z}$ of 43, 45 and 60 (see Figure $4 C$ ). These are the strongest lines in the averaged spectra for region $\mathrm{C}$. The mass spectrum for pure acetic acid [12] looks very similar to the averaged spectra for region $\mathrm{C}$, indicating that the primary species being eluted is acetic acid. Figure 5 presents the spectra resulting from subtraction of the 
acetic acid from Figure 4C (cf. Section 2.2.1), where only carbon dioxide and water remain. These species are probably the tails of regions A and B.

The dominant elution of carbon dioxide, along with the acetic acid and water, can be explained by the presence of $\mathrm{Na}$ carbonate on the glass fiber surface as described above. To clarify the mechanism of carbon dioxide formation (Scheme 1), ${ }^{13} \mathrm{C}$ enriched acetic acid was used as a probe molecule. If the carbon atom in carbon dioxide is coming from the acetic acid itself, carbon dioxide will have an $\mathrm{m} / \mathrm{z}$ of 45 , instead of 44 .

IGC-MS of the same glass fiber dosed with ${ }^{13} \mathrm{C}$ enriched acetic acid at $100{ }^{\circ} \mathrm{C}$ gives similar TIC plot as that in Figure 3. The strongest line of evolving carbon dioxide is $\mathrm{m} / \mathrm{z} 44$. Most significant is the absence of species with $\mathrm{m} / \mathrm{z}$ of 45 (due to the $\mathrm{C}^{13}$ ) in the eluted products. The residual spectrum corresponding to Figure 4, which was obtained by subtraction of the pure ${ }^{13} \mathrm{C}$ enriched acetic acid spectrum, further confirms that the carbon dioxide is not coming from the acetic acid because no signal at $\mathrm{m} / \mathrm{z}$ of 45 is observed. Instead, the carbon dioxide was formed from the reaction of acetic acid with sodium-carbonate already on the surface.

NMR was used to probe the acetic acid surface reactions with boroaluminosilicate fiber and sodium carbonate, and corroborate the IGC-MS and XPS results. Figure 6 shows that when sodium aluminoborosilicate glass fiber is dosed with acetic acid, a different chemical shift is observed than when sodium carbonate is dosed with acetic acid, although the difference is small.

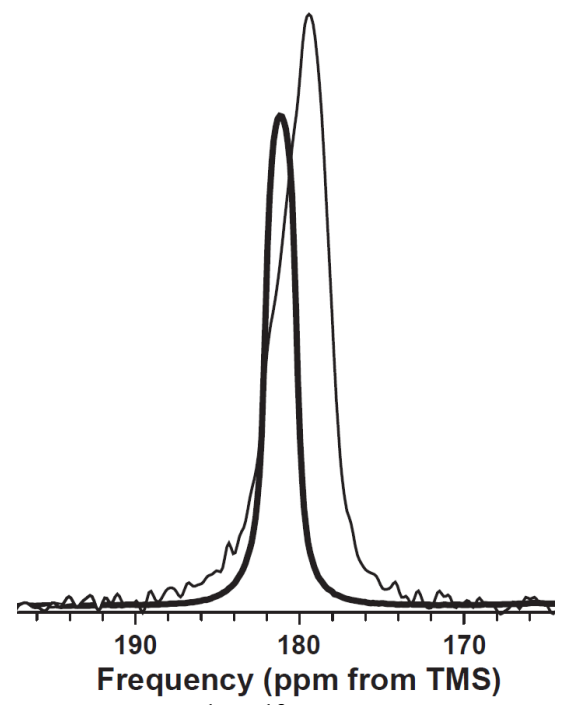

Figure 6. ${ }^{1} \mathrm{H}-{ }^{13} \mathrm{C}$ CP MAS NMR spectra of ${ }^{13} \mathrm{C}$ enriched acetic acid dosed onto freshly pulled sodium aluminoborosilicate glass fiber (thin line) and sodium carbonate (thick line).

The observed chemical shift for the sodium aluminoborosilicate glass fiber dosed with acetic acid is 179 ppm (thin line in Figure 6) which is distinguishable from the observed $181 \mathrm{ppm}$ for the sodium carboxylate (from sodium carbonate powder dosed with acetic acid) (thick line in Figure 6). A closer look at the NMR spectra obtained from the sodium aluminoborosilicate glass fiber dosed with acetic acid (thin line in Figure 6) shows that the peak is not symmetrical. A small shoulder appears on the left side of the peak close to the chemical shift for sodium carbonate dosed with acetic acid. 


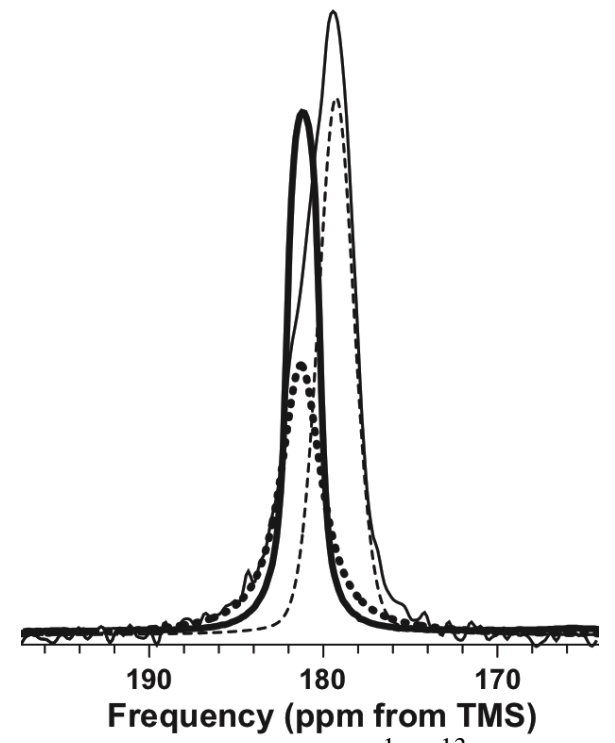

Figure 7. Decoupled ${ }^{1} \mathrm{H}-{ }^{13} \mathrm{C}$ CP MAS NMR spectralines) of ${ }^{13} \mathrm{C}$ enriched acetic acid dosed onto the sodium aluminoborosilicate glass fiber (thin solid line) and onto sodium carbonate (thick solid line).

Through the use of spectral decoupling (see Section 2.2.2), the ${ }^{1} \mathrm{H}-{ }^{13} \mathrm{C}$ CP MAS NMR spectra of ${ }^{13} \mathrm{C}$ enriched acetic acid dosed onto sodium aluminoborosilicate glass fiber (Figure 6) could be resolved into two distinct peaks as shown in Figure 7. The peak with the higher intensity (broken line) has a chemical shift of $179 \mathrm{ppm}$, and the lower intensity peak (dotted line) has a chemical shift of $181 \mathrm{ppm}$. It can now be observed that the peak at $181 \mathrm{ppm}$ (dotted line) corresponds to the reaction of acetic acid with sodium carbonate on the surface to form a distinct sodium carboxylate phase (see Scheme 1 above). The peak at $179 \mathrm{ppm}$ (broken line) is assigned to a sodium carboxylate complex between acetic acid and $\mathrm{a} \equiv \mathrm{Si}-\mathrm{O}^{-} \mathrm{Na}^{+}$site in the glass surface as originally proposed in [10].

To further evaluate this interpretation, the interaction of acetic acid with $=\mathrm{Si}-\mathrm{O}^{-} \mathrm{Na}^{+}$sites in the glass surface was simulated using the Gaussian 03 software package using a gas phase reaction. The use of gas-phase simulation is applicable here since the physisorbed surface water was removed with an in-situ $125^{\circ} \mathrm{C}$ pre-heat prior to experimental NMR analysis. Figure 8A shows the individually optimized (see Section 2.2.4) $\equiv \mathrm{Si}-\mathrm{ONa}{ }^{+}$glass surface site and the acetic acid molecule; these are the reactants. The $\mathrm{C}-\mathrm{O}$ and $\mathrm{C}=\mathrm{O}$ bond lengths of the geometry-optimized acetic acid molecule (Figure 8A) are $1.359 \AA$ and $1.205 \AA$ respectively. The bond length of the hydroxyl species is $0.969 \AA$. These data correspond to literature $[22,25]$. The bond angle of $\mathrm{O}-\mathrm{C}=\mathrm{O}$ is $122.3^{\circ}$ [25]. All of the structural parameters are in excellent agreement with experimentally determined values [26]. The non-bridging oxygen (NBO)-Na distance is $2.148 \AA$, which is smaller than the $2.3 \AA$ value determined elsewhere $[27,28]$. The Si-NBO bond length is $1.565 \AA$. The bridging oxygen (BO)-Si$\mathrm{NBO}$ bond angle of $110.5^{\circ}$ is close to the experimental value of $109.3^{\circ}$ [27]. 


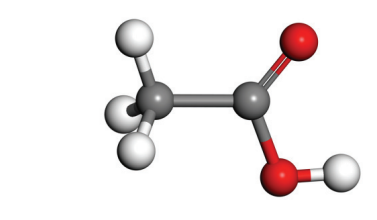

A

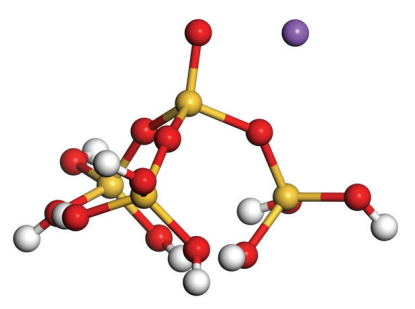

B

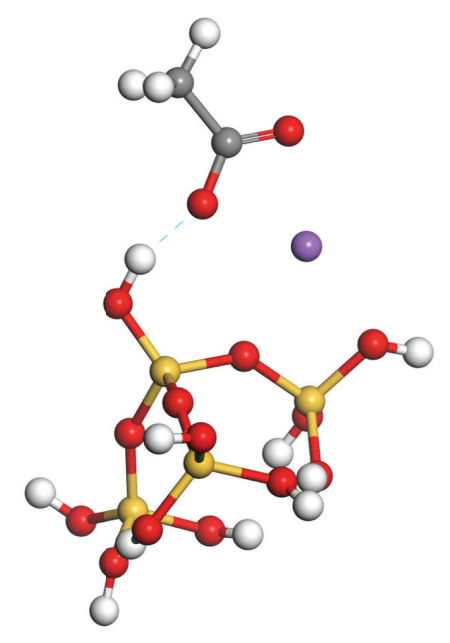

Figure 8. Model of the reaction proposed between acetic acid and the $\equiv \mathrm{Si}^{-} \mathrm{ONa}^{-}$surface site; , A represents reactants, and B represents products; red - oxygen; white - hydrogen; grey - carbon; yellow - silicon; violet - sodium.

Simulation of the reaction product in Figure $8 \mathrm{~B}$ was achieved by placing the acetic acid molecule above the terminal NBO on the $\equiv \mathrm{Si}^{-} \mathrm{O}^{-} \mathrm{Na}^{+}$surface site at a distance of $5 \AA$, and then the cluster group was allowed to optimize. The optimization yielded a stable surface complex through hydrogenation of the NBO and coordination of the sodium ion with the carboxylate anion. The newly-formed carboxylate ion coordinates with the delocalized sodium ion (O-Na distances of 2.277 $\AA$ and $2.289 \AA$ ) to form a sodium carboxylate which deviates from a geometry-optimized sodium carboxylate where the $\mathrm{O}-\mathrm{Na}$ distance is $2.206 \AA$ (the latter optimization was performed with a separate sodium carboxylate molecule not shown in this paper). In addition, a hydrogen bond is formed between the newly-formed silanol and the oxygen on sodium carboxylate with a bond length of $1.640 \AA$, where the short bond length is indicative of strong interaction. Thus, we consider this Na-carboxylate a surface complex, rather than a distinct Na-carboxylate phase, which accounts for its unique NMR signature at 179ppm. The reaction energy calculated for Figure 8B (using Eq. 1) is $140.3 \mathrm{~kJ} / \mathrm{mol}$; it is the sum of five interactions: one hydrogen bond, sodium coordination to carboxylate moieties ( 2 interactions) and a pair of interactions of sodium with one bridging oxygen and one silanol.

\section{Conclusions}

XPS, IGC-MS, NMR and geometry optimization studies were used to better understand the interaction of acetic acid with sodium aluminoborosilicate glass fiber surfaces. XPS analyses revealed that the fiber surfaces contain sodium carbonate weathering products on the surface. The acetic acid which reacts directly with the glass surface, which we call a sodium carboxylate complex, has a different structure (bond distance between oxygen and sodium) resulting in a shift in the NMR peak location, when compared to neat sodium carboxylate formation. This explains the presence of two closely spaced sodium carboxylate peaks in the NMR data (sodium carboxylate via carbonate and the sodium carboxylate glass surface complex). Altogether, it leads to the conclusion that sodium-carbonate on the glass surface will limit the number of adsorption sites for adhesion to the polymer. Since sodium-carbonate is a common weathering product on this sodiumaluminoborosilicate glass composition, its exposure to humid environments should be minimized before application of polymer adhesives containing carboxylic acids. 


\section{Acknowledgments}

This material is based upon work supported by the National Science Foundation under Grant No. CHE-0809657.

\section{References}

[1] F.T. Wallenberger, P.A. Bingham, Fiberglass and Glass Technology - Energy-Friendly Compositions and Applications. Springer US, 2010. doi: 10.1007/978-1-4419-0736-3.

[2] R.K. Iler, The Chemistry of Silica. Solubility, Polymerization, Colloid and Surface Properties, and Biochemistry., Wiley, New York, 1979. doi:10.1002/ange.19800920433.

[3] B. JM, H. Arribart, Attenuated total reflection Fourier transform infrared spectroscopy study of poly(methyl methacrylate) adsorption on a silica thin film : Polymer/surface interactions, Langmuir. 14 (1998) 3716-3719. doi:10.1021/la9703961.

[4] H. Scholze, Proceedings of the Ninth University Conference on Glass Science Glass-water interactions, J. Non. Cryst. Solids. 102 (1988) 1-10. doi:http://dx.doi.org/10.1016/00223093(88)90105-6.

[5] M.M. Curtin Carter, N.S. McIntyre, H.W. King, A. R. Pratt, The aging of silicate glass surfaces in humid air, J. Non. Cryst. Solids. 220 (1997) 127-138. doi:10.1016/s00223093(97)00301-3.

[6] G.I. Rudd, S.H. Garofalini, D.A. Hensley, C.M. Mate, Atomic Force Microscopy and X-ray Photoelectron Spectroscopy Investigation of the Onset of Reactions on Alkali Silicate Glass Surfaces, J. Am. Ceram. Soc. 76 (1993) 2555-2560. doi:10.1111/j.11512916.1993.tb03981.x.

[7] O. Puglisi, A. Torrisi, G. Marletta, XPS investigation of the effects induced by the silanization on real glass surfaces, J. Non. Cryst. Solids. 68 (1984) 219-230. doi:http://dx.doi.org/10.1016/0022-3093(84)90005-X.

[8] J.M. Egan, K.T. Mueller, Detection and Identification of Corrosion Products of Sodium Aluminoborosilicate Glasses by 23Na MQMAS and $1 \mathrm{H} \rightarrow 23 \mathrm{Na}$ CPMAS NMR, J. Phys. Chem. B. 104 (2000) 9580-9586. doi:10.1021/jp992999m.

[9] B.M.J. Smets, T.P.A. Lommen, The Role of Molecular Water in the Leaching of Glass, Phys. Chem. Glasses. 24 (1983) 35-36. 
[10] J.J. Stapleton, D.L. Suchy, J. Banerjee, K.T. Mueller, C.G. Pantano, Adsorption Reactions of Carboxylic Acid Functional Groups on Sodium Aluminoborosilicate Glass Fiber Surfaces, ACS Appl. Mater. Interfaces. 2 (2010) 3303-3309. doi:10.1021/am100730z.

[11] E. Brendlé, E. Papirer, Surface Properties Characterization by Inverse Gas Chromatography (IGC) Applications, in: Powders and Fibers, CRC Press, 2007: pp. 47-122. doi:doi:10.1201/9781420016000.ch2.

[12] NIST Chemistry WebBook. http://webbook.nist.gov/chemistry/.

[13] N. Fairley, http://www. casaxps. com, (2010).

[14] J. Walton, Peak fitting with CasaXPS : a Casa pocketbook, Acolyte Science, Knutsford.

[15] C. Pantano, Chemical Properties of Real and Ideal Glass Surfaces, in: J. McCauley, V. Weiss (Eds.), Mater. Charact. Syst. Perform. Reliab. SE - 7, Springer US, 1986: pp. 127-148. doi:10.1007/978-1-4613-2119-4_7.

[16] A. V Shchukarev, D. V Korolkov, XPS Study of group IA carbonates, Cent. Eur. J. Chem. 2 (n.d.) 347-362. doi:10.2478/BF02475578.

[17] P. Hohenberg, W. Kohn, Inhomogeneous Electron Gas, Phys. Rev. 136 (1964) B864-B871. doi:10.1103/PhysRev.136.B864.

[18] A.D. Becke, Density-functional thermochemistry. III. The role of exact exchange, J. Chem. Phys. 98 (1993) 5648-5652. doi: http://dx.doi.org/10.1063/1.464913

[19] C. Lee, W. Yang, R.G. Parr, Development of the Colle-Salvetti correlation-energy formula into a functional of the electron density, Phys. Rev. B. 37 (1988) 785-789. doi:10.1103/PhysRevB.37.785.

[20] J.A.P. M. J. Frisch, G. W. Trucks, H. B. Schlegel, G. E. Scuseria, M. A. Robb, J. R. Cheeseman, J. A. Montgomery, Jr., T. Vreven, K. N. Kudin, J. C. Burant, J. M. Millam, S. S. Iyengar, J. Tomasi, V. Barone, B. Mennucci, M. Cossi, G. Scalmani, N. Rega, G. A. Pet, Gaussian 03, Revision C.02, Gaussian, Inc., Wallingford CT. (2004).

[21] A. Burneau, F. Genin, F. Quiles, Ab initio study of the vibrational properties of acetic acid monomers and dimers, Phys. Chem. Chem. Phys. 2 (2000) 5020-5029. doi:10.1039/B006230H. 
[22] L. Turi, J.J. Dannenberg, Molecular orbital study of acetic acid aggregation. 1. Monomers and dimers, J. Phys. Chem. 97 (1993) 12197-12204. doi:10.1021/j100149a017.

[23] A. Dkhissi, A. Esteve, M. Djafari-Rouhani, L. Jeloaica, Understanding the Microscopic Structure of SAMs/SiO2 Interfaces in the Presence of Water Using First-Principles Modeling, J. Phys. Chem. C. 112 (2008) 5567-5572. doi:10.1021/jp709697b.

[24] G.I. Rudd, S.H. Garofalini, D.A. Hensley, C.M. Mate, Atomic Force Microscopy and X-ray Photoelectron Spectroscopy Investigation of the Onset of Reactions on Alkali Silicate Glass Surfaces, J. Am. Ceram. Soc. 76 (1993) 2555-2560. doi:10.1111/j.11512916.1993.tb03981.x.

[25] B.P. van Eijck, J. van Opheusden, M.M.M. van Schaik, E. van Zoeren, Acetic acid: Microwave spectra, internal rotation and substitution structure, J. Mol. Spectrosc. 86 (1981) 465-479. doi:http://dx.doi.org/10.1016/0022-2852(81)90294-0.

[26] J.L. Derissen, A reinvestigation of the molecular structure of acetic acid monomer and dimer by gas electron diffraction, J. Mol. Struct. 7 (1971) 67-80. doi:http://dx.doi.org/10.1016/00222860(71)90008-1.

[27] M. Misawa, D.L. Price, K. Suzuki, The short-range structure of alkali disilicate glasses by pulsed neutron total scattering, J. Non. Cryst. Solids. 37 (1980) 85-97. doi:http://dx.doi.org/10.1016/0022-3093(80)90481-0.

[28] J. Biscoe, B.E. Warren, X-Ray Diffraction Study of Soda-Boric Oxide Glass, J. Am. Ceram. Soc. 21 (1938) 287-293. doi:10.1111/j.1151-2916.1938.tb15777.x.

\section{LIST OF FIGURES AND SCHEMES:}

Figure 1. C 1s spectrum of sodium aluminoborosilicate glass fiber.

Figure 2. XPS survey spectrum of sodium aluminoborosilicate glass fiber.

Figure 3. IGC-MS of sodium aluminoborosilicate glass fiber dosed with acetic acid at $100^{\circ} \mathrm{C}$. Lines indicate borders of averaging. 
Figure 4. Mass spectra averaged over the areas A, B and C in Figure 3.

Figure 5. Residual spectrum after subtraction of the acetic acid spectra from Figure 4C (region C).

Scheme 1. Reaction between acetic acid and sodium carbonate weathering products on the fiber surface.

Figure 6. ${ }^{1} \mathrm{H}-{ }^{13} \mathrm{C}$ CP MAS NMR spectra of ${ }^{13} \mathrm{C}$ enriched acetic acid dosed onto freshly pulled sodium aluminoborosilicate glass fiber (thin line) and sodium carbonate (thick line).

Figure 7. Decoupled ${ }^{1} \mathrm{H}_{-}{ }^{13} \mathrm{C}$ CP MAS NMR spectra (dotted and broken lines) of ${ }^{13} \mathrm{C}$ enriched acetic acid dosed onto the sodium aluminoborosilicate glass fiber (thin solid line) and onto sodium carbonate (thick solid line).

Figure 8. Model of the reaction proposed between acetic acid and the $\equiv \mathrm{Si}-\mathrm{O}^{-} \mathrm{Na}^{+}$surface site; , A represents reactants, and B represents products; red - oxygen; white - hydrogen; grey - carbon; yellow - silicon; violet - sodium. 
Na-aluminoborosilicate glass fiber surface dosed with acetic acid

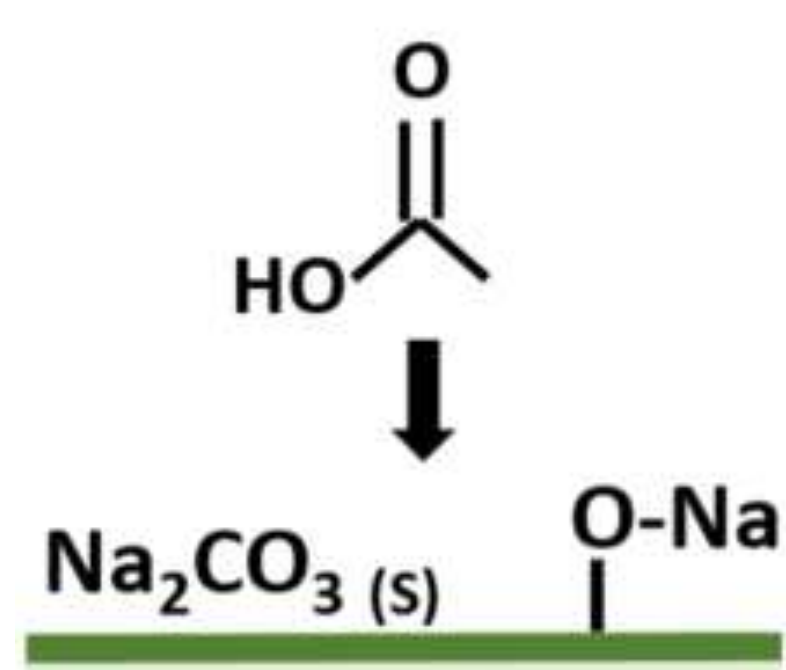

Glass fiber

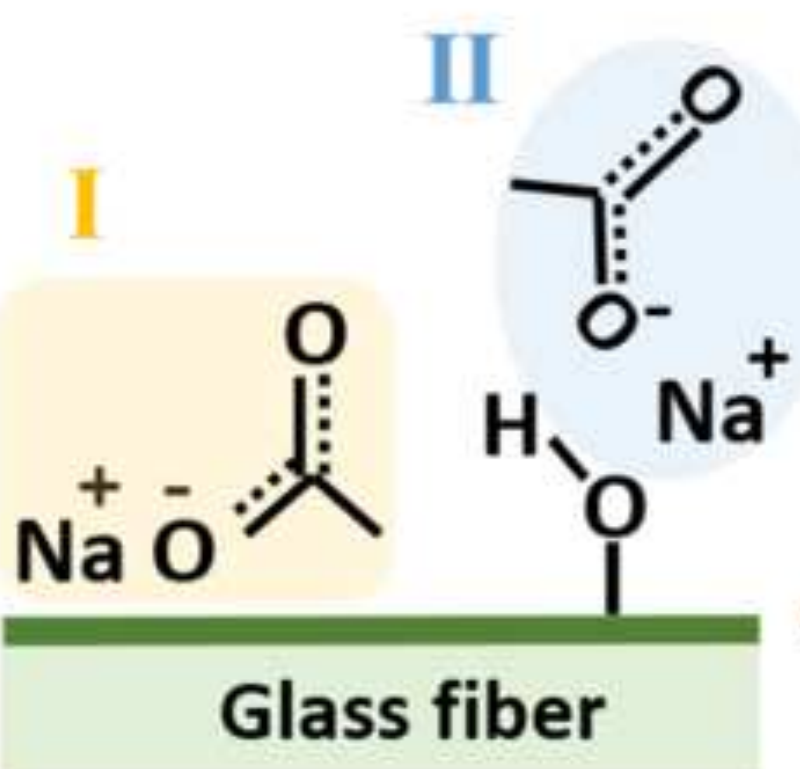

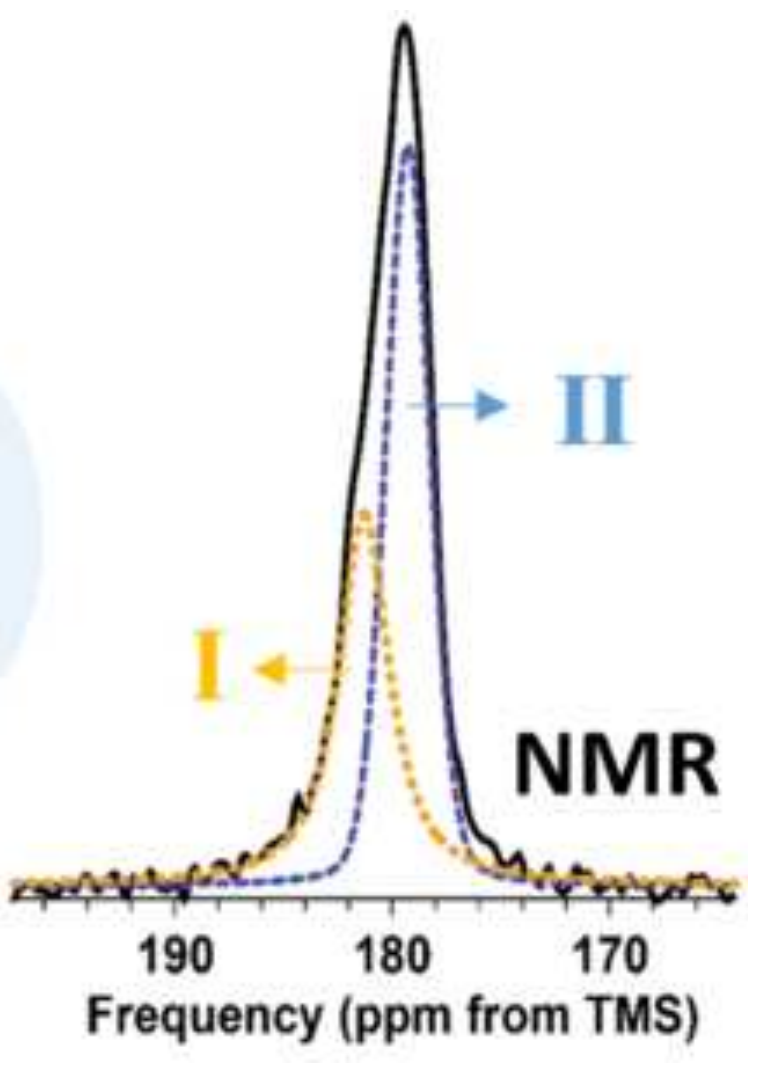

